\title{
SEPARATION OF THE $n$-SPHERE BY AN $(n-1)$-SPHERE
}

BY

JAMES C. CANTRELL(1)

1. Introduction. Let $A$ be the closed spherical ball in $E^{n}$ centered at the origin $O$, and with radius one, $B$ the closed ball centered at $O$ with radius one-half, and $C$ the closed ball centered at $O$ with radius two. The Generalized Schoenflies Theorem states that, if $h$ is a homeomorphism of $\mathrm{Cl}(C-B)$ into $S^{n}$, then $h(\mathrm{Bd} A)$ is tame in $S^{n}$ (the closure of either component of $S^{n}-h(\operatorname{Bd} A)$ is a closed $n$-cell) [5]. One is naturally led to the following question: if $h$ is a homeomorphism of $\mathrm{Cl}(A-B)$ into $S^{n}$, is the closure of the component of $S^{n}-h(\operatorname{Bd} A)$ which contains $h(\operatorname{Bd} B)$ a closed $n$-cell? This question is answered affirmatively by Theorem 1 and should be listed as a corollary to the Generalized Schoenflies Theorem.

Let $D$ be the closed ball in $E^{n}$, centered at $(0,0, \cdots, 0,-1)$ with radius two. Two other types of embeddings of $\operatorname{Bd} A$ in $S^{n}, \quad n>3$, are considered in $\$ 2$, (1) the embedding homeomorphism $h$ can be extended to a homeomorphism of $\mathrm{Cl}(D-B)$ into $S^{n}$ such that the extension is semi-linear on each finite polyhedron in the open annulus Int $(A-B)$, and (2) $h$ can be extended to a homeomorphism of $\mathrm{Cl}(D-A)$ into $S^{n}$ such that the extension is semi-linear in a deleted neighborhood of $(0,0, \cdots, 0,1)$ (see Definition 1$)$. Theorem 4 strongly suggests that, for an embedding of type (1), $h(\operatorname{Bd} A)$ is tame in $S^{n}$. An embedding of this type corresponds to the three dimensional case in which $h(\operatorname{Bd} A)$ is locally polyhedral except at one point.

In $\S 3$, three methods of constructing 3-spheres in $S^{4}$ from 2-spheres in $S^{3}$ are considered: (1) suspension of a 2-sphere in $S^{3}$, (2) rotation of a 2-cell in $S^{3}$ about the plane of its boundary, and (3) capping a cylinder over a 2 -sphere in $S^{3}$. The construction methods in cases (1) and (2) were introduced by Artin [2] and have been used by him and by Andrews and Curtis [1] to construct 2-spheres in $S^{4}$ from 1-spheres in $S^{3}$. Their techniques may be applied directly to establish isomorphism theorems relating the fundamental groups of the complements of the constructed 3-spheres and the fundamental groups of the corresponding complements of the given 2-spheres. Thus, methods (1) and (2) may be used to construct wild (nontame) 3-spheres in $S^{4}$. Method (2) is also used to construct

Presented to the Society, November 17, 1961 under the title Some embeddings of $S^{n-1}$ in $S^{n}$; received by the editors July 24, 1962.

(1) The author wishes to express his appreciation to Professor O. G. Harrold, Jr., for directing the thesis, the principal results of which constitute this paper. This research was supported in part through the NSF-G8239. 
a 3-sphere in $S^{4}$, one complementary domain of which is simply connected but is not an open 4-cell. The third method is used to construct a 3-sphere in $S^{4}$ such that one complementary domain has a closure which is a closed 4-cell, and the other complementary domain is an open 4-cell but its closure is not a closed 4-cell.

2. Some embeddings of $S^{n-1}$ in $S^{n}$. The reader is referred to [5] for the definitions of inverse set and cellular set.

THEOREM 1. Let $h$ be a homeomorphism of $\mathrm{Cl}(A-B)$ into $S^{n}$ and let $G$ be the component of $S^{n}-h(\mathrm{Bd} A)$ which contains $h(\mathrm{Bd} B)$. Then $\mathrm{Cl} G$ is a closed $n$-cell

Proof. Let $G^{\prime}$ be the component of $S^{n}-h(\operatorname{Bd} B)$ which does not contain $h(\mathrm{Bd} A)$. We first observe that $\mathrm{Cl}^{\prime}$ is a cellular subset of $G$. For, if $B_{i}$ is the closed ball in $E^{n}$, centered at $O$ with radius $1 / 2+1 /(i+2), i=1,2, \cdots$, and $G_{i}$ is the component of $S^{n}-h\left(B d B_{i}\right)$ which contains $G^{\prime}$, then, by the Generalized Schoenflies Theorem, $\mathrm{Cl} G_{i}$ is a closed $n$-cell. Furthermore $\mathrm{Cl}_{i+1} \subset G_{i}$ and $\bigcap_{i=1}^{\infty} \mathrm{ClG}_{i}=\mathrm{Cl}^{\prime}$.

Let $g$ be a continuous mapping of $\mathrm{Cl}(A-B)$ onto $A$ such that $\mathrm{Bd} B$ is the only inverse set. Define a mapping $f$ of $\mathrm{Cl} G$ onto $A$ by the equations

$$
\begin{aligned}
& f(x)=g h^{-1}(x), \text { if } x \in \mathrm{Cl} G-G^{\prime}, \\
& f(x)=g(\operatorname{Bd} B), \text { if } x \in G^{\prime} .
\end{aligned}
$$

The mapping $f$ carries $\mathrm{Cl} G$ continuously onto $A$ such that the only inverse set is the cellular subset $\mathrm{Cl}^{\prime}$ of $G$. Thus, by Theorem 2 of [5], $\mathrm{Cl} G$ is a closed $n$-cell.

THEOREM 2. Let $h$ be a homeomorphism of $\mathrm{Cl}(D-B)$ into $S^{n}$ and $G$ be let the component of $S^{n}-h(\operatorname{Bd} A)$ which intersects $h(\mathrm{Bd} D)$. Then $G$ is an open $n$-cell.

Proof. Let $H$ be the component of $S^{n}-h(\operatorname{Bd} A)$ which contains $h(\mathrm{Bd} B)$. By Theorem $1, \mathrm{Cl} H$ is a closed $n$-cell and, hence, there is a homeomorphism $f$ of $A$ onto $\mathrm{Cl} H$ such that $f$ and $h$ agree on $\mathrm{Bd} A$. Define a homeomorphism $\phi$ of $D$ into $S^{n}$ by the equations

$$
\begin{aligned}
& \phi(x)=h(x), \text { if } x \in D-A, \\
& \phi(x)=f(x), \text { if } x \in A .
\end{aligned}
$$

Let $\phi[(0,0, \cdots, 0,1)]=p$ and let $g$ be a continuous mapping of $D$ onto $D$ such that, (1) $g$ is fixed on $\mathrm{Bd} D,(2) g$ is a homeomorphism of $D-A$ onto $D-(0,0, \cdots, 0,1)$, and (3) $g(A)=(0,0, \cdots, 0,1)$. Now define a continuous mapping $\psi$ of $S^{n}$ onto $S^{n}$ by the equations

$$
\begin{array}{ll}
\psi(x)=x, & \text { if } x \in S^{n}-\phi(D), \\
\psi(x)=\phi g \phi^{-1}(x), & \text { if } x \in \phi(D) .
\end{array}
$$


The mapping $\psi$ carries $S^{n}$ onto $S^{n}$, leaves $p$ fixed, and has $\mathrm{Cl} H$ as the only inverse set. Hence, $G$ is carried homeomorphically onto $S^{n}-p$, and is an open $n$-cell.

Let $B_{1}$ be the closed ball in $E^{n}$ which is centered at $O$ and has radius threefourths, and let $L^{\prime}$ be the closed segment of the $x_{n}$-axis from $(0,0, \cdots, 0,3 / 4)$ to $(0,0, \cdots, 0,1)$.

THEOREM 3. Let $h$ be a homeomorphism of $\mathrm{Cl}(D-B)$ into $S^{n}$ and denote $h\left(L^{\prime}\right)$ by $L$ and $h(0,0, \cdots, 0,1)$ by $p$. Let $G$ be the component of $S^{n}-h(\operatorname{Bd} A)$ which intersects $h(\mathrm{Bd} D)$ and let $H$ be the component of $S^{n}-h\left(\mathrm{Bd} B_{1}\right)$ which contains $h(\mathrm{Bd} A)$. Then $\mathrm{Cl} H$ is a closed $n$-cell and $(\mathrm{ClG})-p$ is topologically equivalent to $\mathrm{Cl} H-L$.

Proof. That $\mathrm{Cl} H$ is a closed $n$-cell follows immediately from Theorem 1.

Let $K$ be the component of $S^{n}-h(\mathrm{Bd} D)$ which does not intersect $h(\operatorname{Bd} A)$ and let $g$ be a continuous mapping of $\mathrm{Cl}\left(D-B_{1}\right)$ onto $\mathrm{Cl}(D-A)$ such that (1) $g$ is fixed on $\mathrm{Bd} D$, (2) $g\left(\mathrm{Bd} B_{1}\right)=\mathrm{Bd} A$, and (3) $L^{\prime}$ is the only inverse set under $g$. The mapping $f$ of $\mathrm{Cl} H$ onto $\mathrm{Cl} G$ defined by

$$
\begin{aligned}
& f(x)=x, \quad \text { if } x \in K, \sharp \\
& f(x)=h g h^{-1}(x), \text { if } \quad x \in \mathrm{Cl} H-K,
\end{aligned}
$$

is a continuous mapping of $\mathrm{Cl} H$ onto $\mathrm{Cl} G$ such that the only inverse set is $L$ and $f(L)=p$. Hence, $f$ is a homeomorphism of $\mathrm{Cl} H-L$ onto $\mathrm{Cl} G-p$.

If in Theorem 3 there exists a continuous mapping $k$ of $\mathrm{Cl} H$ onto $\mathrm{Cl} H$ such that $L$ is the only inverse set, then we can state that $\mathrm{Cl} G$ is a closed $n$-cell. In fact, the product mapping $k f^{-1}$ is a homeomorphism of $\mathrm{ClG}$ onto $\mathrm{Cl} H$.

Let us now suppose that $n>3$ and that $h$ is semi-linear on each finite polyhedron of Int $(A-B)$ (we assume a curved decomposition of $E^{n}$ in which $A, B, B_{1}$, and $L^{\prime}$ are polyhedra). Then $h\left(\mathrm{Bd} B_{1}\right)$ is a polyhedron and $L$ is locally polyhedral except at $p$. Let $\varepsilon>0$ be such that $S(\varepsilon, p) \subset H$ and use Lemma 2 of [6] to obtain a homeomorphism $\phi$ of $S^{n}$ onto $S^{n}$ such that $\phi$ is fixed outside $S(\varepsilon, p)$ and $\phi(L)$ is polyhedral. Let $q$ be the endpoint of $L$ which lies on $\operatorname{Bd} H$ and let $Q$ be a polyhedral $n$-cell in $\mathrm{Cl} H$ such that $q \in \mathrm{Bd} Q, \phi(L)-q \subset \operatorname{Int} Q$, and $Q$ has a subdivision isomorphic to a subdivision of a simplex (see [7, Lemma 5.3]). Let $\psi$ be a semilinear homeomorphism of $Q$ onto a simplex $R$. The $\operatorname{arc} \psi \phi(L)$ is then polyhedral in $R$ and, together with the linear segment $\overline{\psi \phi(q) \psi \phi(p)}$, from $\psi \phi(q)$ to $\psi \phi(p)$, bounds a polyhedral 2-cell which, except for $\psi \phi(q)$, lies in the interior of $R$. Lemma 3 of [9] is then applied to obtain a homeomorphism $\eta$ of $R$ onto $R$ such that $\eta$ is fixed on $\operatorname{Bd} R$ and carries $\psi \phi(L)$ onto $\overline{\psi \phi(q) \psi \phi(p)}$. It is then easy to find a continuous mapping $\theta$ of $R$ onto $R$ such that $\theta$ is fixed on $\operatorname{Bd} R, \theta(\overline{\psi \phi(q) \psi \phi(p)})$ $=\psi \phi(q)$, and $\overline{\psi \phi(q) \psi \phi(p)}$ is the only inverse set. The mapping $k$, defined by

$$
\begin{array}{ll}
k(x)=\phi(x), & \text { if } x \notin \phi^{-1}(Q), \\
k(x)=\psi^{-1} \theta \eta \psi \phi(x), & \text { if } x \in \phi^{-1}(Q),
\end{array}
$$


is a continuous mapping of $\mathrm{ClH}$ onto $\mathrm{Cl} H$ such that $L$ is the only inverse set. Thus, we have the following theorem.

THEOREM 4. Let $n>3$ and let $h$ be a homeomorphism of $\mathrm{Cl}(D-B)$ into $S^{n}$. If $h$ is semi-linear on each finite polyhedron of $\operatorname{Int}(A-B)$, then $h(\operatorname{Bd} A)$ is tame in $S^{n}$.

The semi-linear condition in Theorem 4 is used only to shrink $L$ to a boundary point of $\mathrm{ClH}$. It seems that one should be able to remove this condition and retain the conclusion, since the local embedding at each point $t$ of $L$, different from $p$, is as "nice" as the local embedding of an interval at one of its points. In fact, for each $t \in L$, different from $p$ one can find a homeomorphism $h_{t}$ of $S^{n}$ onto itself such that the subarc $L_{t}$ of $L$ from $q$ to $t$ is carried onto a linear segment.

Definition 1. Let $h$ be a homeomorphism of $\mathrm{Cl}(D-A)$ into $S^{n}$. If there exists a neighborhood $N$ of $(0,0, \cdots, 0,1)$ in $E^{n}$ such that $h$ is semi-linear on each finite polyhedron of Int $(D-A) \cap N$, then we say that $h$ is semi-linear on a deleted neighborhood of $(0,0, \cdots, 0,1)$.

THEOREM 5. Let $n>3$ and $h$ a homeomorphism of $\mathrm{Cl}(D-A)$ into $S^{n}$ such that $h$ is semi-linear on a deleted neighborhood of $(0,0, \cdots, 0,1)$. If $G$ is the component of $S^{n}-h(\mathrm{Bd} A)$ which intersects $h(\mathrm{Bd} D)$, then $\mathrm{Cl} G$ is a closed $n$-cell.

Proof. The technique of proof used here is that used by Mazur in [8].

Let $D_{1}$ be a cell, obtained from $D$ by a slight contraction on $E^{n}$ toward $(0,0, \cdots, 0,1)$, such that $\left(B d D_{1}\right)-(0,0, \cdots, 0,1)$ is contained in $D-A$. Let $G_{1}$ and $G_{2}$, respectively, be the components of $S^{n}-h\left(\operatorname{Bd} D_{1}\right)$ and $S^{n}-h(\operatorname{Bd} D)$ which are contained in $G$. We now observe that $\mathrm{Cl}_{1}$ is homeomorphic to $\mathrm{ClG}$. For, if $g$ is a homeomorphism of $E^{n}$ onto itself which is fixed on $\operatorname{Bd} D$ and carries $\operatorname{Bd} D_{1}$ onto $\operatorname{Bd} A$, then the mapping $\phi$ defined by

$$
\begin{array}{ll}
\phi(x)=x, & \text { if } x \in G_{2}, \\
\phi(x)=h g h^{-1}(x), & \text { if } x \in \mathrm{Cl}\left(G_{1}-G_{2}\right),
\end{array}
$$

carries $\mathrm{Cl}_{1}$ homeomorphically onto $\mathrm{ClG}$. This suggests the following observation: if one attaches a copy of $\mathrm{Cl} G_{1}$ to $\mathrm{Cl}\left(D_{1}-A\right)$ along $\mathrm{Bd} D_{1}$ with $h^{-1}$, the set thus obtained is equivalent to $\mathrm{ClG}_{1}$ (it is simply $\mathrm{ClG}$ ). This will be used to show that $\mathrm{Cl}_{1}$ is a closed $n$-cell, and hence that $\mathrm{Cl} G$ is a closed $n$-cell.

Let $N$ be a neighborhood of $(0,0, \cdots, 0,1)$ such that $h$ is semi-linear on $\operatorname{Int}(D-A) \cap N$. Let $S$ be an $n$-simplex in $\operatorname{Cl}\left(D_{1}-A\right) \cap N$, such that $(0,0, \cdots, 0,1)$ is a vertex of $S$ and let $K=S^{n}-h(S)$. By Theorem $4, \mathrm{Cl} K$ is a closed $n$-cell. Let $H=S^{n}-\mathrm{Cl} G$, then $\mathrm{Cl} K$ can be realized by taking $P=\mathrm{Cl}\left(D_{1}-A\right)-$ Int $S$ and attaching $\mathrm{Cl} H$ to $P$ along $\mathrm{Bd} A$ with $h^{-1}$, and attaching $\mathrm{Cl}_{1}$ to $P$ along $\operatorname{Bd} D_{1}$ with $h^{-1}$. The set $P$ is a closed $n$-cell (the closure of the exterior of $S$ ) with the interiors of two $n$-cells, sharing a common boundary point with $\operatorname{Bd} S$, 
removed. The cell obtained from $P$ by attaching $\mathrm{Cl}_{1}$ and $\mathrm{Cl} H$ to the interior boundary spheres of $P$ with $h^{-1}$ will be denoted by $\bar{P}$.

Let $F$ be the part of the solid unit ball in $E^{n}$ centered at $(0,0, \cdots, 0,1,0)$, determined by $x_{n} \geqq 0$. Let $\left\{q_{i}\right\}_{i=0}^{\infty}$ be a sequence of points in the intersection of the plane $x_{1}=x_{2}=\cdots=x_{n-2}=0$ and $\mathrm{Bd} F$ such that, if $q_{i}=(0,0, \cdots$, $\left.a_{(n-1) i}, a_{n i}\right)$, then $a_{(n-1) 0}=2, a_{n 0}=0$, the $a_{(n-1) i}$ converge monotonically to zero, and $a_{n i}>0$ for $i>0$. We then section $F$ into a countable number of $n$-cells by projecting the $(n-2)$-plane $x_{n}=x_{n-1}=0$ onto each of the $q_{i}$. The section determined by $q_{i-1}$ and $q_{i}$ is denoted by $C_{i}$. We then delete from $C_{i}$ the interior of a cell $C_{i}^{\prime}$, similar in shape to $C_{i}$ and, except for the boundary point $(0,0, \cdots, 0,0)$, contained in the interior of $C_{i}$. Any two adjacent sections then form a copy of $P$, and are labeled $P_{i}, P_{i}^{\prime}$, as in Figure 1 . Notice that $P_{i}$ and $P_{i}^{\prime}$ have $w_{2 i}=\mathrm{Bd}_{2 i}^{\prime}$ in common, and $P_{i}^{\prime}$ and $P_{i+1}$ have $w_{2 i+1}=\mathrm{Bd}_{2 i+1}^{\prime}$ in common.

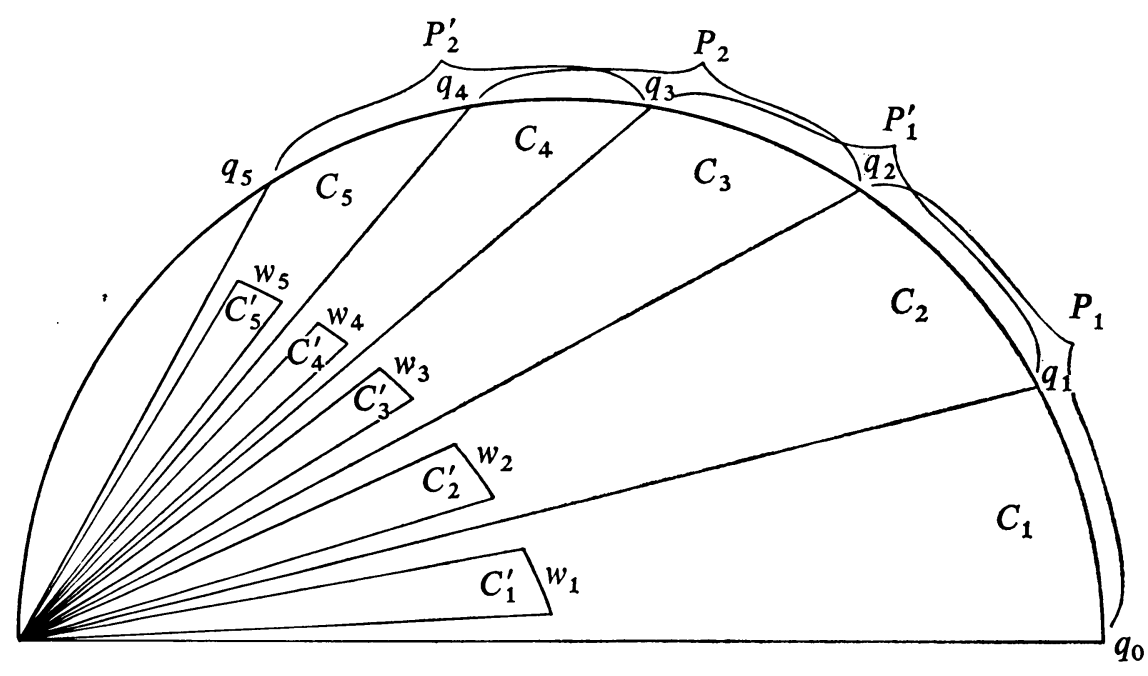

FIGURE 1

Let $\phi_{i}$ be a homeomorphism of $P_{i}$ onto $P_{i}^{\prime}$ which leaves $w_{2 i}$ fixed and carries $w_{2 i-1}$ onto $w_{2 i+1}$. Let $\psi_{i}$ be a homeomorphism of $P_{i}^{\prime}$ onto $P_{i+1}$ which leaves $w_{2 i+1}$ fixed and carries $w_{2 i}$ onto $w_{2 i+2}$. We identify $P_{1}$ with $P$, with $w_{1}$ identified with $\mathrm{Bd} D_{1}$ and $w_{2}$ identified with $\mathrm{Bd} A$. The sets $\mathrm{Cl}_{1}$ and $\mathrm{Cl} H$ are then sewn to $P$ along $w_{1}$ and $w_{2}$, respectively, with $h^{-1}$. The resulting $n$-cell is denoted by $\bar{P}_{1}$. The sets $\mathrm{ClG}_{1}$ and $\mathrm{Cl} H$ are then sewn into alternate holes bounded by $w_{2 i+1}$ and $w_{2 i+2}$ by the attaching homeomorphisms

$$
\begin{aligned}
& \phi_{i} \cdots \phi_{2} \phi_{1} h^{-1}: \mathrm{Bd} G_{1} \rightarrow w_{2 i+1}, \\
& \psi_{i} \cdots \psi_{2} \psi_{1} h^{-1}: \operatorname{Bd} H \rightarrow w_{2 i+2} .
\end{aligned}
$$


The sets thus obtained from the $P_{i}$ and $P_{i}^{\prime}$ are denoted by $\bar{P}_{i}$ and $\bar{P}_{i}^{\prime}$ and we set $F_{1}=\bigcup_{i=1}^{\infty} \bar{P}_{i}$.

Since $\phi_{1}$ is the identity on $w_{2}$, we can extend $\phi_{1}$ to a homeomorphism of $\bar{P}_{1}$ onto $\bar{P}^{\prime}$, and conclude that $\bar{P}_{1}^{\prime}$ is also a closed $n$-cell. In a similar manner we extend $\psi_{i}$ to a homeomorphism of $\bar{P}_{i}^{\prime}$ onto $\bar{P}_{i+1}$ and extend $\phi_{i}$ to a homeomorphism of $\bar{P}_{i}$ onto $\bar{P}_{i}^{\prime}$. It then follows that each $\bar{P}_{i}$ and each $\bar{P}_{i}^{\prime}$ is a closed $n$-cell.

We now observe that $F_{1}$ is a closed $n$-cell. We map the boundary of $C_{2 i-1} \cup C_{2 i}$ onto the boundary of $\bar{P}_{i}$ with the identity homeomorphism. Since $C_{2 i-1} \cup C_{2 i}$ and $\bar{P}_{i}$ are $n$-cells, this homeomorphism between their boundaries can be extended to a homeomorphism between the cells. These extensions for $i=1,2, \cdots$, yield a homeomorphism of $F$ onto $F_{1}$.

We next observe that $F_{1}$ is a copy of $\mathrm{Cl}\left(D_{1}-A\right)$ with $\mathrm{Cl}_{1}$ sewn along one of the boundary spheres. This can be established by showing that $F_{1}$, with $G_{1}$ removed from $\bar{P}_{1}$, is homeomorphic to $F$, with Int $C_{1}^{\prime}$ removed. Let $\lambda$ be the identity mapping on $C_{1}$ - Int $C_{1}^{\prime}$ and on $\operatorname{Bd}\left(C_{2 i} \cup C_{2 i+1}\right), i=1,2, \cdots$. Since $C_{2 i} \cup C_{2 i+1}$ and $\bar{P}_{i}^{\prime}$ are closed $n$-cells and $\lambda$ restricts to a homeomorphism between their boundaries, $\lambda$ can be extended over their interiors. These extensions over each of the $C_{2 i} \cup C_{2 i+1}$ yield the desired homeomorphism.

We have seen that $F_{1}$ can first be viewed as a closed $n$-cell, and secondly as $\mathrm{Cl}_{1}$ sewn into a boundary sphere of a copy of $\mathrm{Cl}\left(D_{1}-A\right)$. We previously observed that a set of the second type is equivalent to $\mathrm{Cl} G_{1}$. Hence $\mathrm{Cl}_{1}$, or equivalently $\mathrm{Cl} G$, is a closed $n$-cell, and Theorem 5 is proved.

If one were able to remove the semi-linear condition in Theorem 4 , then the semi-linear condition in Theorem 5 could also be removed( $\left.{ }^{2}\right)$. In this general form Theorem 5 would imply that a wild $(n-1)$-sphere is $S^{n}, n>3$, must be "knotted" at more than one point, and that such simple examples of wild spheres as the Fox-Artin examples [3] for $n=3$ do not exist in the higher dimensional spaces.

\section{Some 3-spheres in $S^{4}$.}

Definition 2. In $E^{4}$ we take coordinates $x_{1}, x_{2}, x_{3}, x_{4}$ and let $E^{3}$ be described by $x_{4}=0$. Let $a=(0,0,0,1)$ and $b=(0,0,0,-1)$. For a set $A$ in $E^{3}$ the suspension of $A$ in $E^{4}$ is the join of $A$ and $a \cup b$, and is denoted by Susp $A$.

The proof of Theorem 1 of [1] may be used directly to prove the following theorem.

THEOREM 6. Let $S$ be a 2-sphere in $E^{3}$ and $K=\operatorname{Susp} S$. Let $A_{1}$ and $A_{2}$ be the bounded and unbounded components of $E^{3}-S$ respectively, and $B_{1}, B_{2}$ the corresponding components of $E^{4}-K$. Then the injection homomorphism $i_{j}: \pi_{1}\left(A_{j}\right) \rightarrow \pi_{1}\left(B_{j}\right), j=1,2$, is an onto isomorphism.

(2) Added in proof. After this paper was sent to press the author was able to remove the semi-linear conditions in Theorem s4 and 5. These results, together with certain generalizations, will appear in print at a later date. 
Let $E_{+}^{3}=\left\{\left(x_{1}, x_{2}, x_{3}, 0\right) \in E^{4} \mid x_{3} \geqq 0\right\}$ and let $P$ be the plane $x_{3}=x_{4}=0$. For $x=\left(x_{1}, x_{2}, x_{3}, 0\right)$ and $0 \leqq t<2 \pi$ we set $R_{t}(x)=\left(x_{1}, x_{2}, x_{3} \cos t, x_{3} \sin \mathrm{t}\right)$, and for a subset $M$ of $E_{3}^{+}$we set $R(M)=\left\{R_{t}(x) \mid x \in M, 0 \leqq t<2 \pi\right\}$. For a subset $N$ of $E^{4}$ we set $R^{-1}(N)=\left\{y \in E_{+}^{3} \mid R_{t}(y) \in N\right.$ for some $\left.0 \leqq t<2 \pi\right\}$.

If $M$ is a 2-cell in $E_{+}^{3}$ such that $M \cap P=\operatorname{Bd} M=d$, and $D$ is the bounded component of $P-d$, then the proof of Theorem 3 of [1] may be used to establish the following theorem.

TheOREM 7. Let $A_{1}$ and $A_{2}$ be the bounded and unbounded components, respectively, of $E_{+}^{3}-(M \cup D)$ and let $B_{1}, B_{2}$ be the corresponding components of $E^{4}-R(M)$. Then $\pi_{1}\left(A_{i}\right) \approx \pi_{1}\left(B_{i}\right), i=1,2$.

In [3] there are examples of 2-spheres in $S^{3}$ such that one complementary domain has a nontrivial fundamental group. Elementary modifications of these examples will give 2-spheres in $S^{3}$ such that the fundamental group of either complementary domain is nontrivial. These examples, together with Theorem 6 or Theorem 7, give the existence of 3-spheres in $S^{4}$ such that either one or both complementary domains have nontrivial fundamental groups. In passing, we observe one difference between the spheres Susp $S$ and $R(M)$. Associated with each exceptional point $p \in S$ there will be an arc, Susp $p$, of exceptional points on Susp $S$, and for each exceptional point $p \in M$ there will be a simple closed curve, $R(p)$, of exceptional points on $R(M)$.

We now use the rotation of a disk about $P$ to construct a 3-sphere in $S^{4}$, one complementary domain of which is simply connected but is not an open 4-cell. Let us first embed the 2-sphere $S$, discussed as Example 3.2 in [3], in

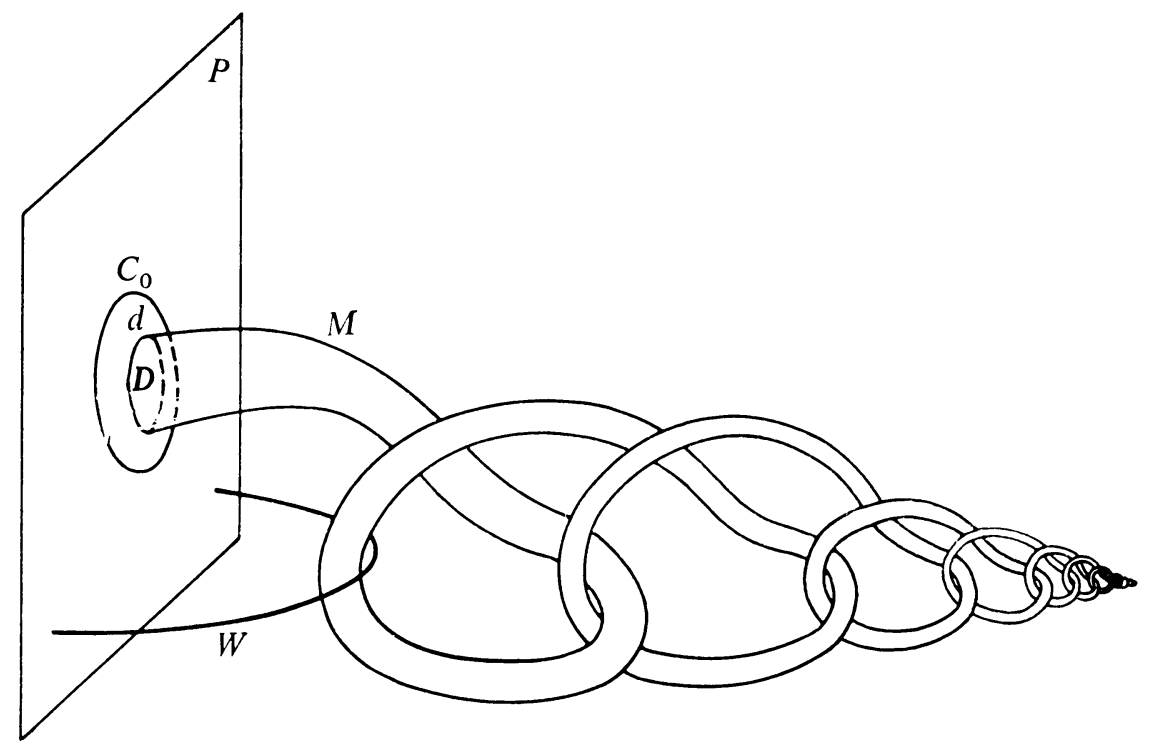

FIGURE 2 


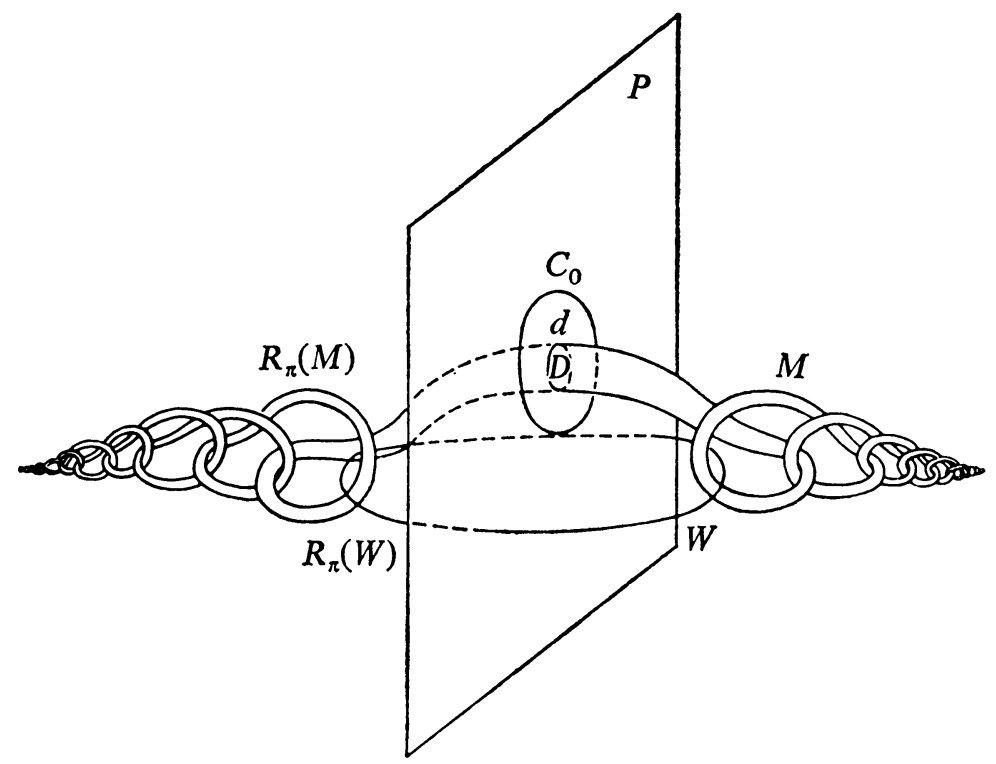

FIGURE 3

$E_{+}^{3}$ as indicated in Figure 2. The sphere $S$ is to intersect $P$ in a 2-cell $D$ and $\mathrm{Cl}(S-D)$ is denoted by $M$. If $L$ is the arc described as Example 1.3 in [3], the proof in [3] that $E^{3}-L$ is simply connected may be used directly to show that $A_{2}$ (the exterior of $S$ in $E_{+}^{3}$ ) is simply connected. Hence, by Theorem 7, $B_{2}$ (the exterior of $R(M)$ in $E^{4}\left(S^{4}\right)$ ) is simply connected.

The cross section $M \cup R_{\pi}(M)$ of $R(M)$ is shown in Figure 3.

Let $A_{2}^{\prime}$ denote the exterior of $M \cup R_{\pi}(M)$ in $E^{3}$. It is shown in [3, Example 1.3] that $C_{0}$ cannot be contracted to a point in $A_{2}^{\prime}-\left[W \cup R_{\pi}(W)\right]$. This fact is now used to show that $R(W)$ is contained in no closed 4-cell subset of $B_{2}$ whose complement in $B_{2}$ is simply connected. Hence, $B_{2}$ is not an open 4-cell.

Suppose that such a 4-cell $J$ did exist. Choose the base point for computing $\pi_{1}\left(B_{2}-J\right)$ in $P$ and so close to $d$ that there is a path $c_{0}$ in $\left(B_{2}-J\right) \cap P$ which cannot be contracted to a point in $A_{2}^{\prime}-\left[W \cup R_{n}(W)\right]$. Let $E$ be a unit disk in $E^{2}$ with boundary $e$, and let $h$ be a continuous mapping of $e$ onto $c_{0}$. Since $\pi_{1}\left(B_{2}-J\right)$ is trivial, there exists an extension $H$ of $h$ which carries $E$ into $B_{2}-J$. We then follow $H$ by $R^{-1}$ and obtain a singular 2-cell, $R^{-1} H(E)$, in $A_{2}-R^{-1}(J)$ which is bounded by $c_{0}$. Since $A_{2}-R^{-1}(J) \subset A_{2}-W$, we see that $c_{0}$ can be contracted to a point in $A_{2}-W$ and hence in the larger set $A_{2}^{\prime}-\left[W . \cup R_{\pi}(W)\right]$. This contradiction establishes the desired conclusion.

We now describe a third method for constructing $(n-1)$-spheres in $S^{n}$ and refer to this method as capping a cylinder.

In $E^{n}$ we again take coordinates $x_{1}, x_{2}, \cdots, x_{n}$ and let $E^{n-1}$ be described by $x_{n}=0$. 
LEMMA 1. Let $S$ be an $(n-2)$-sphere in $E^{n-1}$ with the bounded and unbounded components of $E^{n-1}-S$ denoted by $A_{1}$ and $A_{2}$, respectively. If $\mathrm{Cl} A_{2}$ (compactified at infinity) is a closed (n-1)-cell, then $\{\mathrm{S} \times[0,1]\} \cup\left\{\mathrm{Cl} A_{1} \times[1]\right\}$ is a closed ( $n-1)$-cell.

Proof. Let $h$ be a homeomorphism of $\mathrm{Cl} A_{2}$ onto a standard unit ball $B$ in $E^{n-1}$. Let $S_{1}=\mathrm{Bd} B$ and let $S_{2}$ be the sphere concentric with $S_{1}$ and with radius one-half. Then $h^{-1}\left(S_{2}\right)$ is an $(n-2)$-sphere in $E^{n-1}$ and if $C$ is the component of $E^{n-1}-h^{-1}\left(S_{2}\right)$ which contains $A_{1}$, then, by Theorem $1, \mathrm{ClC}$ is a closed $(n-1)$ cell. We now observe that $\mathrm{Cl} C$ consists of a closed annulus with $\mathrm{Cl} A_{1}$ sewn along one boundary component and is, therefore, a copy of $\{S \times[0,1]\} \cup\left\{\mathrm{Cl} A_{1} \times[1]\right\}$.

THeOREM 8. Let $S, A_{1}$, and $A_{2}$ be as in Lemma 1. If $\mathrm{Cl} A_{2}$ (compactified at infinity) is a closed (n-1)-cell, then $\{S \times[-1,1]\} \cup\left\{\mathrm{Cl} A_{1} \times[-1]\right\} \cup\left\{\mathrm{Cl} A_{1} \times[1]\right\}$ is an $(n-1)$-sphere.

Proof. By Lemma 1, each of $\{S \times[-1,0]\} \cup\left\{\mathrm{Cl} A_{1} \times[-1]\right\}$ and $\{S \times[0,1]\} \cup\left\{\mathrm{Cl} A_{1} \times[1]\right\}$ is a closed $n$-cell. These cells meet along their common boundary sphere $S$, and hence their union is an $(n-1)$-sphere.

We now consider a 2 -sphere $S$, locally polyhedral except at a single point, in $E^{3}\left(S^{3}\right)$ such that the bounded complementary domain $A_{1}$ is an open 3-cell, $\mathrm{Cl} A_{1}$ is not a closed 3-cell, the unbounded complementary domain (compactified at infinity) is an open 3-cell, and $\mathrm{Cl} A_{2}$ is a closed 3-cell. The assertion is that the 3-sphere

$$
T=\{S \times[-1,1]\} \cup\left\{\mathrm{Cl} A_{1} \times[1]\right\} \cup\left\{\mathrm{Cl} A_{1} \times[-1]\right\}
$$

is embedded in $S^{4}$ such that, if $B_{1}$ and $B_{2}$, respectively, are the components of $S^{4}-T$ which contain $A_{1}$ and $A_{2}$, then $B_{1}$ is an open 4-cell, $\mathrm{Cl}_{1}$ is not a closed 4-cell, and $\mathrm{Cl} B_{2}$ is a closed 4-cell.

Since $B_{1}$ is the product of the open 3-cell $A_{1}$ and the open interval $(-1,1)$, $B_{1}$ is an open 4-cell. If $\mathrm{Cl}_{1}=\mathrm{Cl} A_{1} \times[-1,1]$ were a closed 4-cell, a theorem due to Bing [4] would imply that $\mathrm{Cl} A_{1}$ is a closed 3-cell. Thus contradicting our assumption on the embedding of $S$ in $S^{3}$.

We now show that $\mathrm{Cl}_{2}$ is a closed 4-cell by constructing a homeomorphism $f: T \times[0,1 / 2] \rightarrow \mathrm{Cl}_{2}$ such that $f_{0}(y)=f(y, 0)=y$ for each $y \in T$ and then applying Theorem 1. Since $\mathrm{Cl} A_{2}$ is a closed 3-cell, there exists a homeomorphism $h: S \times[0,1 / 2] \rightarrow \mathrm{Cl}_{2}$ such that $h_{0}(x)=h(x, 0)=x$ for each $x \in S$. For $y \in T$, let $x$ be the point of $\mathrm{Cl} A_{1}$ which lies under $y(y=(x, t)$ for some $t \in[-1,1])$. We define $f$ by the following equations:

(1) $f_{r}(y)=(x, 1+r)$, if $y=(x, 1)$;

(2) $f_{r}(y)=(x,-1-r), \quad$ if $y=(x,-1)$;

(3) $f_{r}(y)=\left(h_{r}(x), t\right), \quad$ if $x \in S$ and $-1+r<t<1-r$; 
(4) $f_{r}(y)=\left(h_{(1-t)}(x), 2 t-(1-r)\right)$, if $x \in S$ and $1-r \leqq t \leqq 1$;

(5) $f_{r}(y)=\left(h_{(1-t)}(x), 2 t-(r-1)\right)$, if $x \in S$ and $-1 \leqq t \leqq-1+r$.

The continuity of $f$ follows rather quickly from the definition of $f$ in terms of the continuous mapping $h$ and a set of linear equations. The one-to-one property of $f$ depends principally on the fact that each arc $f_{r}(x \times[0,1])$ must lie over the $\operatorname{arc} L_{x}=\left\{h_{s}(x) \mid s \in[0,1 / 2]\right\}$ and that $L_{x_{1}}$ and $L_{x_{2}}$ intersect if and only if $x_{1}=x_{2}$.

\section{BIBLIOGRAPHY}

1. J. J. Andrews and M. L. Curtis, Knotted 2-spheres in the 4-sphere, Ann. of Math. (2) 70 (1959), 565-571.

2. E. Artin, Zur Istopie zweidimensionaler Flächen im $\mathbf{R}_{4}$, Abh. Math. Sem. Univ. Hamburg 4 (1925), 174-177.

3. E. Artin and R. H. Fox, Some wild cells and spheres in three dimensional space, Ann. of Math. (2) 49 (1948), 979-990.

4. R. H. Bing, A set is a 3-cell if its cartesian product with an arc is a 4-cell, Proc. Amer. Math. Soc. 12 (1961), 13-19.

5. M. Brown, A proof of the Generalized Schoenflies Theorem, Bull. Amer. Math. Soc. 66 (1960), 74-76.

6. J. C. Cantrell and C. H. Edwards, Almost locally polyhedral curves in Euclidean $n$-space, Trans. Amer. Math. Soc. 107 (1963), 451-457.

7. V. K. A. M. Gugenheim, Piecewise linear isotopy and embedding of elements and spheres. I, Proc. London Math. Soc. 3 (1953), 29-53.

8. B. Mazur, On embeddings of spheres, Bull. Amer. Math. Soc. 65 (1959), 59-65.

9. E. C. Zeeman, Unknotting spheres, Ann. of Math. (2) 72 (1960), 350-361.

UNIVERSITY OF TENNESSEE,

KNOXVILLE, TENNESSEE 